\title{
AN END-FAITHFUL SPANNING TREE COUNTEREXAMPLE
}

\author{
PAUL SEYMOUR AND ROBIN THOMAS
}

(Communicated by Jeffry N. Kahn)

\begin{abstract}
We find an infinitely-connected graph in which every spanning tree has a 2-way infinite path. This disproves Halin's well-known "end-faithful spanning tree" conjecture and also disproves a recent conjecture of Šrán̆.
\end{abstract}

\section{INTRODUCTION}

A ray in a graph $G$ is a 1-way infinite path. (In this paper, graphs may be infinite and may have loops or multiple edges.) Two rays $R_{1}, R_{2}$ in $G$ are parallel if for every finite $X \subseteq V(G)$, the unique component of $G \backslash X$ that has infinite intersection with $R_{1}$ also has infinite intersection with $R_{2} .(G \backslash X$ is the graph obtained from $G$ by deleting $X$.) Parallelness is an equivalence relation, and its equivalence classes are called the ends of $G$. These were first investigated by Halin [4], who proposed the following "end-faithful spanning tree conjecture," which we shall disprove.

(1.1) Conjecture. In every connected graph $G$ there is a spanning tree $T$ such that each end of $G$ includes a unique end of $T$.

Halin [3, 4] proved that (1.1) holds if $G$ is countable and that it holds if $G$ does not contain $K_{\aleph_{0}}$. (We denote by $K_{\kappa}$ the complete graph with $\kappa$ vertices, when $\kappa$ is a cardinal. A graph $G$ contains a graph $H$ if some subgraph of $G$ is isomorphic to a subdivision of $H$-that is, a graph obtained from $H$ by replacing its edges by internally disjoint paths.) However, we shall see that (1.1) is false in general. A counterexample has independently been obtained by $\mathrm{C}$. Thomassen [9].

Let us say that $G$ is infinitely-connected if $V(G)$ is infinite and $G \backslash X$ is connected for every finite $X \subseteq V(G)$. Since an infinitely-connected graph has a unique end, a consequence of (1.1) would be the following:

(1.2) Conjecture. In every infinitely-connected graph there is a spanning tree with a unique end.

We shall give a counterexample to (1.2) and hence to (1.1).

Received by the editors January 23, 1990.

1980 Mathematics Subject Classification (1985 Revision). Primary 05C05, 05C75. 
The following conjecture was proposed at a recent (1989) conference in Cambridge by Širán. (A tree is rayless if it has no ray.)

(1.3) Conjecture. Let $G$ be a connected graph, and suppose that for every ray $R$ there is a vertex $v$ such that, for every finite $X \subseteq V(G)-\{v\}$, the component of $G \backslash X$ containing $v$ has infinite intersection with $R$. Then $G$ has a rayless spanning tree.

Šrán [8] proved this for countable graphs $G$, but we shall see that it is false in general. Since every infinitely-connected graph satisfies the hypothesis of (1.3), a consequence of (1.3) would be the following:

(1.4) Conjecture. In every infinitely-connected graph there is a rayless spanning tree.

Our counterexample to (1.1) and (1.2) is also a counterexample to (1.4) and hence to (1.3). (Indeed, we shall show that a graph satisfies (1.2) if and only if it satisfies (1.4).)

Let $T_{\aleph_{1}}$ be the tree in which every vertex has valency $\aleph_{1}$. We shall show the following:

(1.5). There is an infinitely-connected graph $G$ with $|V(G)|=2^{\aleph_{0}}$ such that every spanning tree contains $T_{\aleph_{1}}$.

In particular, every spanning tree has $\geq \aleph_{1}$ ends, contrary to (1.2) and (1.4). We shall also show that

(1.6). There is an infinitely-connected graph $G$ with $|V(G)|=2^{\aleph_{0}}$, which does not contain $K_{\aleph_{1}}$, such that every spanning tree contains $T_{\aleph_{1}}$.

We do not know whether (1.5) remains true with $2^{\aleph_{0}}$ replaced by $\aleph_{1}$. However, we shall see that the existence of $G$ as in (1.6) with $|V(G)|=\aleph_{1}$ rather than $2^{\aleph_{0}}$ is independent of ZFC (Zermelo-Fraenkel set theory together with the axiom of choice). Finally, we shall show that

(1.7). Every infinitely-connected graph which does not contain $T_{\aleph_{1}}$ has a rayless spanning tree.

Before we begin the main proofs, let us see the equivalence of (1.2) and (1.4). (1.8). Let $G$ be an infinitely-connected graph. Then $G$ has a rayless spanning tree if and only if it has a spanning tree with exactly one end.

Proof. Let $T$ be a rayless spanning tree, and let $R$ be a ray. Extend $R$ to a spanning tree $T^{\prime}$ of $R \cup T$; then $T^{\prime}$ has exactly one end (for any ray not parallel to $R$ includes a ray disjoint from $R$, and so contains a ray in $T$ ). For the converse, let $T$ be a spanning tree with only one end, and let $R$ be a ray of $T$, with $V(R) \neq V(G)$. Choose $v \in V(G)-V(R)$. Since $G$ is infinitely-connected, there are infinitely many finite paths of $G$ from $v$ to $V(R)$, mutually disjoint except for $v$. Let the union of these paths be $F$, and extend $F$ to a spanning 
tree $T^{\prime}$ of $F \cup T$. Suppose that $R^{\prime}$ is a ray of $T^{\prime}$. Since $F$ is connected and includes no ray, and $F \cup R^{\prime}$ includes no circuit, it follows that $R^{\prime}$ includes a ray disjoint from $F$, so we may assume that $R^{\prime}$ is disjoint from $F$. Hence $R^{\prime} \subseteq T$, and so $R, R^{\prime}$ are parallel in $T$. Since $T$ is a tree, it follows that $R^{\prime \prime}=R \cap R^{\prime}$ is a ray. But $R^{\prime} \cap F$ is null, and $\left(V(R)-V\left(R^{\prime \prime}\right)\right) \cap V(F)$ is finite, and yet $V(R) \cap V(F)$ is infinite, a contradiction. Thus $T^{\prime}$ is rayless, as required.

We state a stronger form of (1.4) for countable graphs that we shall apply later. We omit the proof, which is easy. Let $G$ be a graph, let $v \in V(G)$ and let $\left\{P_{n}\right\}_{n=1}^{\infty}$ be a collection of finite paths in $G$, each with at least one edge, with one endpoint $v$ and otherwise disjoint. The tree $R=P_{1} \cup P_{2} \cup \cdots$ is called an $\omega$-star in $G$ with center $v$.

(1.9). Every countable, infinitely-connected graph has a spanning $\omega$-star with center any specified vertex.

\section{THE COUNTEREXAMPLE}

A hypergraph is a pair $(V, M)$, where $V$ is a set and $M$ is a set of subsets of $V$. Two hypergraphs $(V, M),\left(V^{\prime}, M^{\prime}\right)$ are isomorphic if there is a bijection $\alpha: V \rightarrow V^{\prime}$ mapping $M$ to $M^{\prime}$ (and $\alpha$ is an isomorphism).

Let $(V, M)$ be a hypergraph such that

(i) every member of $M$ has cardinality $\aleph_{0}$,

(ii) for every partition $\left(X_{1}, X_{2}, \ldots\right)$ of $V$ into countably many sets, some $X_{i}$ includes some member of $M$, and

(iii) $|V|,|M| \geq \aleph_{1}$.

(In fact, condition (iii) is implied by (i) and (ii), as the reader may verify.) For example, we could take $V=\aleph_{1}$ and let $M$ be the set of all countable subsets of $V$. We shall specify $(V, M)$ later.

Let $\Sigma$ be the set of all finite sequences of members of $M$. If $\sigma=\left(\mu_{1}, \ldots\right.$, $\left.\mu_{k}\right) \in \Sigma$ and $\mu \in M$, we denote the sequence $\left(\mu_{1}, \ldots, \mu_{k}, \mu\right) \in \Sigma$ by $\sigma+(\mu)$. For each $\sigma \in \Sigma$, let $\left(V_{\sigma}, M_{\sigma}\right)$ be a hypergraph isomorphic to $(V, M)$ such that $V_{\sigma} \cap V_{\sigma^{\prime}}=\varnothing$ for all distinct $\sigma, \sigma^{\prime} \in \Sigma$, and let $\alpha_{\sigma}: V \rightarrow V_{\sigma}$ be an isomorphism. For each $\mu \in M$, we denote $\left\{\alpha_{\sigma}(v): v \in \mu\right\}$ by $\mu_{\sigma}$; thus, $\mu_{\sigma} \in M_{\sigma}$.

Let $G$ be the graph with $V(G)=\bigcup\left(V_{\sigma}: \sigma \in \Sigma\right)$, in which $u, v \in V(G)$ are adjacent if $u \in \mu_{\sigma}$ and $v \in V_{\sigma^{\prime}}$, for some $\sigma \in \Sigma$ and $\mu \in M$, where $\sigma^{\prime}=\sigma+(\mu)$. We shall show that every spanning tree of $G$ contains $T_{\aleph_{1}}$.

We shall need the following lemma, which is very similar to a result of Laver [5] and which can be proved similarly (we omit the proof).

(2.1). Let $T$ be a tree which does not contain $T_{\aleph_{1}}$, and let $v_{0} \in V(T)$. There is a function $\phi$ assigning an ordinal $\phi(v)$ to each $v \in V(T)$, such that

(i) if $u, v \in V(T)$ are adjacent and $u$ lies on the path of $T$ between $v_{0}$ and $v$, then $\phi(v) \leq \phi(u)$, and 
(ii) for all $u \in V(T)$ the set of all $v \in V(T)$ as in (i) with $\phi(v)=\phi(u)$ has cardinality $\leq \aleph_{0}$.

(2.2). With $G$ as defined earlier, every spanning tree of $G$ contains $T_{\aleph_{1}}$.

Proof. Suppose that $T$ is a spanning tree of $G$ not containing $T_{\aleph_{1}}$. Choose $v_{0} \in V_{\sigma_{0}}$, where $\sigma_{0}$ is the null sequence, and for $u, v \in V(G)$, let us say that $u$ is before $v$ if $u$ lies on the path of $T$ between $v_{0}$ and $v$.

For each $\sigma \in \Sigma$, let $B_{\sigma}$ denote $\bigcup V_{\sigma^{\prime}}$, the union being taken over all $\sigma^{\prime} \in \Sigma$ of which $\sigma$ is an initial subsequence. Let us say that $u \in V(G)$ dominates $\sigma \in \Sigma$ if $u$ is before every $v \in B_{\sigma}$, and let us say that $u \in V(G)$ is big if it dominates some $\sigma \in \Sigma$. Thus, $v_{0}$ is big. Let $\phi$ be as in (2.1), and choose a big vertex $u \in V(G)$ with $\phi(u)$ minimum.

(1) There are only countably many $v \in V(G)$ such that $u$ is before $v$ and $\phi(u) \leq \phi(v)$.

For $\phi(u)=\phi(v)$ for every such $v$. Let $X$ be the set of all such $v$, and let $R$ be the minimal subtree of $T$ with $X \subseteq V(R)$. Then $u \in V(R)$ and $u$ lies before every other vertex of $R$. Since every vertex of $R$ lies on a path between $u$ and some vertex $v$ of $X$, and the $\phi$-values on such a path do not increase (by $(2.1)(\mathrm{i})$ ), and $\phi(v)=\phi(u)$, it follows that every vertex of this path belongs to $X$, and in particular $V(R)=X$. Thus every vertex of $R$ has valency $\leq \aleph_{0}$ (by $(2.1)(\mathrm{ii}))$, and so $|V(R)| \leq \aleph_{0}$. This proves (1).

Since $u$ dominates some $\sigma \in \Sigma$ and hence dominates all extensions of $\sigma$ in $\Sigma$, we may choose $\sigma \in \Sigma$ such that $u$ dominates $\sigma$ and $u \notin B_{\sigma}$. Since $M$ is uncountable, there are uncountably many 1 -term extensions $\sigma^{\prime}$ of $\sigma$, and the corresponding sets $B_{\sigma^{\prime}}$ are mutually disjoint. Thus by (1) we may choose $\sigma \in \Sigma$ such that, in addition, there is no $v \in B_{\sigma}$ with $\phi(u) \leq \phi(v)$. Choose $\mu \in M$, and let $\sigma^{\prime}=\sigma+(\mu)$.

Let $S$ be the minimal subtree of $T$ with $\mu_{\sigma} \subseteq V(S)$. Since $\mu_{\sigma}$ is countable, it follows that so is $V(S)$. For each $s \in V(S)$, let $X_{s}$ be the set of all $v \in V_{\sigma^{\prime}}$ such that there is a path of $T$ between $s$ and $v$ with no vertex in $V(S)$ except $s$. Thus $\left(X_{s}: s \in V(S)\right)$ is a partition of $V_{\sigma^{\prime}}$ into countably many sets, and so there exists $s \in V(S)$ and $\mu^{\prime} \in M$ such that $\mu_{\sigma^{\prime}}^{\prime} \subseteq X_{s}$. Let $\sigma^{\prime \prime}=\sigma^{\prime}+\left(\mu^{\prime}\right)$. We claim that

$$
s \text { dominates } \sigma^{\prime \prime} \text {. }
$$

For let $v \in B_{\sigma^{\prime \prime}}$, and let $P$ be the path of $T$ between $v_{0}$ and $v$. Since $v_{0} \in$ $V_{\sigma_{0}}$, it follows that $V(P) \cap \mu_{\sigma^{\prime}}^{\prime} \neq \varnothing$. Let $x \in V(P) \cap \mu_{\sigma^{\prime}}^{\prime}$. Since $V(P) \cap \mu_{\sigma} \neq \varnothing$, and hence $V(P) \cap V(S) \neq \varnothing$, it follows that $P$ includes the unique minimal path of $T$ between $x$ and $V(S)$. Since $x \in \mu_{\sigma^{\prime}}^{\prime} \subseteq X_{s}$, it follows that $s \in V(P)$. Thus $s$ is before $v$, as required.

$$
s \in B_{\sigma} .
$$


For since $X_{s}$, is infinite and $X_{s} \cap V(S) \subseteq\{s\}$, there exists $v \in X_{s}-V(S)$. Thus $v \in V_{\sigma^{\prime}}$. Let $P$ be the path of $T$ between $v$ and $s$. Since no vertex of $P$ is in $V(S)$ except $s$ (because $\left.v \in X_{s}\right)$ and $\mu_{\sigma} \subseteq V(S)$, it follows that $V(P) \cap \mu_{\sigma} \subseteq\{s\}$, and so either $s \in B_{\sigma^{\prime}}$ or $s \in \mu_{\sigma}$. In either case, $s \in B_{\sigma}$ as required.

Now we chose $\sigma$ such that there is no $v \in B_{\sigma}$ with $\phi(u) \leq \phi(v)$, and so from (3) we deduce that $\phi(s)<\phi(u)$. But $s$ is big by (2), contrary to the choice of $u$. This completes the proof.

So far we have not specified the collection $M$ of sets used in the construction of the graph $G$ above. As we saw before, we can take $V=\aleph_{1}$ and $M$ to be the collection of all countable subsets of $V$, and the graph $G$ we construct satisfies (1.5). This proves (1.5). However, that graph contains $K_{\aleph_{1}}$, and in view of Halin's theorem that every counterexample to (1.1) and (1.2) contains $K_{\aleph_{0}}$, it is natural to ask if every counterexample also contains $K_{\aleph_{1}}$. The answer is no, as we shall see by a more complicated choice of $M$.

A well-founded tree is a poset $T=(V(T), \leq)$, such that for every pair $t, t^{\prime} \in V(T)$ their infimum $\inf \left(t, t^{\prime}\right)$ exists, and for every $t \in V(T)$ the set $\left\{t^{\prime} \in V(T): t^{\prime} \leq t\right\}$ is well ordered by $\leq$. It follows that every well-founded tree $T$ has a minimum element, called the root of $T$ and denoted by $\operatorname{root}(T)$. Let $V$ be the set of all transfinite sequences of distinct positive integers, and let us say for such sequences $s_{1}, s_{2}$, that $s_{1} \leq s_{2}$ if $s_{1}$ is an initial segment of $s_{2}$. It was shown in the Ph.D. thesis of D. Kurepa (and later, independently, by R. Laver; see [1]) that the well-founded tree $(V, \leq)$ cannot be partitioned into countably many antichains, and $|V|=2^{\aleph_{0}}$. Let $M$ be the collection of all infinite chains of $(V, \leq)$; we claim that $(V, M)$ satisfies the requirements at the start of this section. Let $\left(X_{1}, X_{2}, \ldots\right)$ be a partition of $V$; we must show that some $X_{i}$ includes an infinite chain of $(V, \leq)$. Suppose not; then each $X_{i}$ can be partitioned into countably many antichains, and hence so can $V$, a contradiction. Thus $(V, M)$ satisfies our requirements, and so the corresponding graph $G$ satisfies (2.2). Moreover, it follows from the results of [6] that $G$ does not contain $K_{\aleph_{1}}$. This proves (1.6).

\section{AN INDEPENDENCE RESULT}

We have seen that (1.2) and (1.4) are

(i) true for all countable graphs and for all graphs that do not contain $K_{\aleph_{0}}$,

(ii) false for a graph with $2^{\aleph_{0}}$ vertices which does not contain $K_{\aleph_{1}}$.

What about graphs with $\aleph_{1}$ vertices? We do not know whether such a graph can satisfy (1.5) (and hence falsify (1.2) and (1.4)), but for (1.6) we have an independence result. Let us consider the truth of the following statement:

(3.1). In every infinitely-connected graph $G$ with $|V(G)|=\aleph_{1}$ that does not contain $K_{\aleph_{1}}$, there is a rayless spanning tree. 
We shall see that (3.1) is independent of ZFC. For we observe from (1.6) that

(3.2). If the continuum hypothesis holds, then (3.1) is false.

On the other hand, Baumgartner, Malitz, and Reinhardt [2] proved that the following statement is consistent with ZFC (although not with the continuum hypothesis):

(3.3). If $(V, \leq)$ is a well-founded tree with $|V| \leq \aleph_{1}$, and every chain of $(V, \leq)$ has order type $<\omega_{1}$, then $V$ may be partitioned into countably many antichains.

In the rest of this section we shall prove that (3.3) implies (3.1). If $T=$ $(V(T), \leq)$ is a well-founded tree and $t_{1}, t_{2} \in V(T)$, we say that $t \in V(T)$ is between $t_{1}$ and $t_{2}$ if $\inf \left(t_{1}, t_{2}\right) \leq t$, and either $t \leq t_{1}$, or $t \leq t_{2}$. We say that $t_{1}$ is a predecessor of $t_{2}$ if $t_{1} \leq t_{2}$ and there is no $t \in V(T)-\left\{t_{1}, t_{2}\right\}$ such that $t_{1} \leq t \leq t_{2}$. A well-founded tree-decomposition of a graph $G$ is a pair $(T, W)$, where $T$ is a well-founded tree and $W=\left(W_{t}: t \in V(T)\right)$ is a collection of sets such that

(W1) $\bigcup\left(W_{t}: t \in V(T)\right)=V(G)$, and every edge of $G$ has both its ends in some $W_{t}$;

(W2) if $t^{\prime}$ is between $t$ and $t^{\prime \prime}$ in $T$, then $W_{t} \cap W_{t^{\prime \prime}} \subseteq W_{t^{\prime}}$; and

(W3) if $t$ has no predecessor, then $W_{t} \supseteq \bigcup_{t^{\prime}<t} \cap_{t^{\prime} \leq t^{\prime \prime}<t} W_{t^{\prime \prime}}$.

We need the following structure theorem [6, (2.7)].

(3.4). Let $G$ be an infinitely-connected graph with $|V(G)| \leq \aleph_{1}$ that does not contain $K_{\aleph_{1}}$. Then there exists a well-founded tree-decomposition $(T, W)$ of $G$ such that

(i) $|V(T)| \leq \aleph_{1}$,

(ii) every chain of $T$ has order type $<\omega_{1}$, and

(iii) $W_{t}$ induces a countable-infinitely connected graph in $G$ for every $t \in$ $V(T)$.

(3.5). If (3.3) holds, then (3.1) holds.

Proof. Let $G$ be an infinitely-connected graph with $|V(G)|=\aleph_{1}$ which does not contain $K_{\aleph_{1}}$, and let $(T, W)$ be a well-founded tree-decomposition of $G$ as in (3.4).

(1) For every $v \in V(G)$, there exists a unique minimal element $t \in V(T)$ with $v \in W_{t}$.

For there exists at least one such $t$ by (W1), and the minimal one is unique by the existence of infima and (W2).

For $v \in V(G)$, the element $t \in V(T)$ as in (1) will be denoted by $t(v)$. From (i) and (ii) of (3.4) and (3.3), $V(T)$ can be partitioned into countably many antichains, say $A_{1}, A_{2}, \ldots$. 
Let $t_{0}$ be the root of $T$. An ideal of $T$ is a subset $S \subseteq V(T)$ such that $t_{0} \in S$ and such that $s \in S$ for every $s \in V(T)$ with $s \leq t$ for some $t \in S$. Choose $v_{0} \in W_{t_{0}}$. A sprout is a triple $(S, R, \rho)$, where $S$ is an ideal of $T$, $R$ a tree of $G$ with $\bigcup_{s \in S} W_{s}=V(R)$, and $\rho$ is a function from $V(R)$ into $\{1,2, \ldots\}$ such that the following hold:

$$
\text { If } v \neq v_{0} \text { is between } v_{0} \text { and } v^{\prime} \text { in } R \text {, then } \rho(v) \geq \rho\left(v^{\prime}\right) \text {, and }
$$
the inequality is strict unless $t(v)=t\left(v^{\prime}\right)$,

The set $\{v \in V(R): t(v)=t$ and $\rho(v) \leq i\}$ is finite for every $t \in V(T)$ and every $i \in\{1,2, \ldots\}$,

For every $v \in V(R)$ with $t(v) \in A_{i}, \rho(v) \geq i$.

We first prove the following:

If $(S, R, \rho)$ is a sprout, $t \in V(T)$, and $i \geq 1$ is an integer, then $\left\{v \in W_{t} \cap V(R): \rho(v) \leq i\right\}$ is finite.

We prove (5) by transfinite induction. The statement follows from (3) if $t=t_{0}$, so let $t \in V(T)-\left\{t_{0}\right\}$, let $i \geq 1$ be an integer, and assume that (5) holds for all $t^{\prime}<t$. There exists $t_{1} \in V(T)$ with $t_{1}<t$ such that $t^{\prime} \notin A_{1} \cup \cdots \cup A_{i}$ for every $t^{\prime} \in V(T)$ with $t_{1}<t^{\prime}<t$. Hence

$$
\begin{aligned}
\left\{v \in W_{t} \cap V(R): \rho(v) \leq i\right\} \subseteq & \left\{v \in W_{t} \cap V(R): \rho(v) \leq i, t(v) \leq t_{1}\right\} \\
& \cup\left\{v \in W_{t} \cap V(R): \rho(v) \leq i, t(v)=t\right\},
\end{aligned}
$$

by (4) and (W2). The first set is a subset of $\left\{v \in W_{t_{1}} \cap V(R): \rho(v) \leq i\right\}$, and hence is finite by the induction hypothesis, and the second one is finite by (3). This proves $(5)$.

There exists at least one sprout.

For let $S=\left\{t_{0}\right\}$, and let $R=P_{1} \cup P_{2} \cup \cdots$ be the spanning $\omega$-star of the subgraph of $G$ induced by $W_{t_{0}}$ with center $v_{0}$, which exists by (1.9), and (iii) of (3.4). Let $\rho: V(R) \rightarrow\{1,2, \ldots\}$ be such that if $\rho(v)=i$, then $v \in V\left(P_{i}\right)$. Then $(S, R, \rho)$ is a sprout, as desired.

We order sprouts by saying that $(S, R, \rho) \leq\left(S^{\prime}, R^{\prime}, \rho^{\prime}\right)$ if $S \subseteq S^{\prime}, R$ is a subtree of $R^{\prime}$, and $\rho(r)=\rho^{\prime}(r)$ for every $r \in V(R)$. By Zorn's lemma, there exists a maximal sprout $(S, R, \rho)$.

$$
S=V(T) \text {. }
$$

For suppose not; then there exists $t \in V(T)-S$, minimal. Let $k$ be such that $t \in A_{k}$, let $F=\bigcup_{s \in S} W_{s}$, and let $u_{k}, u_{k+1}, \ldots$ be all the vertices of $W_{t}-F$. Since $W_{t} \cap V(R)=W_{t} \cap F$, and since $W_{t} \cap F$ is infinite (because $W_{t} \cap F$ separates the infinite sets $W_{t}$ and $W_{t_{0}}$ and $G$ is infinitely-connected) we deduce by repeated application of (5) and (iii) of (3.4) that there exist mutually disjoint finite paths $P_{k}, P_{k+1}, \ldots$ such that for every $i \geq k, V\left(P_{i}\right) \subseteq W_{t}, P_{i}$ 
has as one end some $w \in W_{t} \cap F$ with $\rho(w)>i$, and has no other vertex in $F$, and $u_{i} \in \bigcup_{k \leq i^{\prime} \leq i} V\left(P_{i^{\prime}}\right)$. In particular,

$$
W_{t}-F \subseteq \bigcup_{i \geq k} V\left(P_{i}\right) \subseteq W_{t}
$$

Let $S^{\prime}=S \cup\{t\}$, let $R^{\prime}=R \cup P_{k} \cup P_{k+1} \cup \cdots$, and let $\rho^{\prime}$ be defined by

$$
\rho^{\prime}(r)= \begin{cases}\rho(r) & \text { if } r \in V(R) \\ i & \text { if } r \in V\left(P_{i}\right)-V(R) .\end{cases}
$$

Then $\left(S^{\prime}, R^{\prime}, \rho^{\prime}\right)$ is a sprout, contradicting the maximality of $(S, R, \rho)$. This proves (7).

From (7) and (W1) we deduce that $R$ is a spanning tree of $G$, and from (2) and (3) it follows that $R$ is rayless.

We deduce that both (3.1) and its negation are relatively consistent with ZFC, and hence (3.1) is independent of ZFC.

\section{EXCLUdING THE $\aleph_{1}$-TREE}

Our final objective is to prove (1.7), which we restate:

(4.1). Every infinitely-connected graph which does not contain $T_{\aleph_{1}}$ has a rayless spanning tree.

If $X \subseteq V(G)$, an $X$-flap is the vertex set of a component of $G \backslash X$. We shall need the following, a consequence of [7, Theorem (2.3)].

(4.2). Let $G$ be a graph that does not contain $T_{\aleph_{1}}$. For each $X \subseteq V(G)$ with $|X| \leq \aleph_{0}$, let $\beta(X)$ be a union of $X$-flaps such that if $X \subseteq Y \subseteq V(G)$ and $|Y| \leq \aleph_{0}$, then $\beta(X)$ includes precisely those $X$-flaps which meet $\beta(Y)$. Then $\beta(\varnothing)=\varnothing$.

Proof of (4.1). Let us say that $X \subseteq V(G)$ is good if $X \subseteq V(T)$ for some rayless tree $T$ of $G$ (not necessarily a spanning tree).

(1) Every countable subset of $V(G)$ is good.

For let $X \subseteq V(G)$ be countable. Then $X \subseteq V(H)$ for some countable subgraph $H$ of $G$ which is infinitely-connected, and by (1.9) $H$ has a spanning $\omega$-star. Hence $X$ is good.

$$
\begin{aligned}
& \text { If } X \subseteq V(G) \text { is good and } C_{i} \quad(i \in I) \text { are good } X \text {-flaps, then } \\
& X \cup \cup\left(C_{i}: i \in I\right) \text { is good. }
\end{aligned}
$$

For let $T$ be a rayless tree of $G$ with $X \subseteq V(T)$, and for each $i \in I$ let $T_{i}$ be a rayless tree with $C_{i} \subseteq V\left(T_{i}\right)$. Since $G$ is connected, we may assume that each $T_{i}$ intersects $T$. For each $i \in I$, choose $S_{i} \subseteq T_{i}$ minimal such that $S_{i} \cup T$ is connected and $C_{i} \subseteq V\left(S_{i} \cup T\right)$. Then each component of $S_{i}$ has exactly one vertex in $V(T)$, and $V\left(S_{i} \cup T\right)=C_{i} \cup V(T)$. Thus, $T \cup \cup\left(S_{i}: i \in I\right)$ is a rayless tree of $G$, and its vertex set includes $X \cup \cup\left(C_{i}: i \in I\right)$, as required. 
For each $X \subseteq V(G)$ with $|X| \leq \aleph_{0}$, let $\beta(X)$ be the union of all $X$-flaps that are not good.

If $X \subseteq Y \subseteq V(G)$ and $|Y| \leq \aleph_{0}$, then $\beta(X)$ includes just those $X$-flaps that meet $\beta(Y)$.

For, if an $X$-flap $C$ meets a $Y$-flap $D \subseteq \beta(Y)$, then $D \subseteq C$; and so $C$ is not good, since $D$ is not good, and hence $C \subseteq \beta(X)$. Conversely, let $C$ be an $X$-flap with $C \cap \beta(Y)=\varnothing$. Let the $Y$-flaps included in $C$ be $C_{i} \quad(i \in I)$. Since $C \cap \beta(Y)=\varnothing$, each $C_{i}$ is good, and so by (1) and (2) $Y \cup \bigcup\left(C_{i}: i \in I\right)$ is good. Hence $C$ is good, since $C \subseteq Y \cup \cup\left(C_{i}: i \in I\right)$, and so $C \notin \beta(X)$. This proves (3).

From (3) and (4.2), we deduce that $\beta(\varnothing)=\varnothing$. Hence $G$ has a rayless spanning tree, as required.

\section{ACKNOWLEDGMENT}

We are grateful to R. Diestel for pointing out an error in an earlier version of this paper.

\section{REFERENCES}

1. J. E. Baumgartner, Results and independence proofs in combinatorial set theory, $\mathrm{Ph} . \mathrm{D}$. Thesis, University of California, 1970.

2. J. E. Baumgartner, J. Malitz, and W. Reinhardt, Embedding trees in the rationals, Proc. Nat. Acad. Sci. USA 67 (1970), 1748-1753.

3. R. Halin, Simplicial decompositions of infinite graphs, in Advances in Graph Theory (B. Bollobás, ed.), North-Holland, 1978.

4. __ Über unendliche Wege in Graphen, Math. Ann. 157 (1964), 125-137.

5. R. Laver, Better-quasi-ordering and a class of trees, Studies in foundations and combinatorics, Adv. Math. Suppl. Stud., vol. 1, 1978, pp. 31-48.

6. N. Robertson, P. D. Seymour, and R. Thomas, Excluding subdivisions of infinite cliques, submitted.

7. P. D. Seymour and R. Thomas, Excluding infinite trees, Trans. Amer. Math. Soc. (to appear).

8. J. Šrán̆, Coterminal forests and spanning trees in infinite graphs, Proceedings of the Cambridge Conference on Infinite Graphs 1989, submitted.

9. C. Thomassen, Infinite connected graphs with no end-preserving spanning trees, manuscript, 1990.

Bellcore, 445 South Street, Morristown, New Jersey 07960

Dimacs Center, Hill Center, Busch campus, Rutgers University, New Brunswick, NEW JERSEY 08903

School of Mathematics, Georgia Institute of Technology, Atlanta, Georgia 30332 